\title{
CONSOLIDACIÓN DE UNA PRÁCTICA INTEGRADORA EN FISIOLOGÍA HUMANA
}

Consolidation of an integrative practice in human physiology

Consolidação de uma prática inclusiva em fisiologia humana

\section{Ildefonso Alvear-Ordenes}

Departamento de Ciencias Biomédicas, Universidad de León, España. Teléfono: +34987442039. Correo electrónico: ialvor@unileon.es

\section{Resumen}

El objetivo de esta experiencia fue diseñar una práctica que integrara en una sola sesión, los diferentes sistemas fisiológicos estudiados hasta ese momento, en la asignatura de ‘Fisiología y Fisiopatología’, del primer año del Grado en Enfermería. Por tanto, la práctica denominada "Pruebas funcionales de diferentes sistemas", debía servir como una experiencia integradora. Para ello, se utilizó un protocolo de ejercicio continuo, que permitió medir la adaptación al estrés en un período relativamente corto de tiempo. Este tipo de protocolo es similar a los que utiliza el deportista en sus entrenamientos y permite observar los cambios adaptativos al estrés en diferentes variables fisiológicas (Frecuencia cardíaca y respiratoria, ácido láctico, glucemia, riego subdérmico, temperatura corporal central y periférica, etc.). Al alumno se le evalúa la capacidad de estructurar y analizar gráficos. Se concluye que esta práctica permite demostrar diversos conceptos de integración y adaptación fisiológica.

Palabras clave: fisiología integrativa; biología integrativa; fisiología del ejercicio

\begin{abstract}
The objective of this experience was to design a practice that integrated in single session, the different physiological systems studied until that moment, in the subject of 'Physiology and Pathophysiology', of the first year of the Degree in Nursing. Therefore, the practice called "Functional tests of different systems", should serve as an integrating experience. A continuous exercise protocol was used to measure the adaptation to stress in a relatively short period of time. This type of protocol is similar to that used by the athlete in his workouts and allows the observation of adaptive changes to stress in


different physiological variables (heart rate and respiratory rate, lactic acid, blood glucose, subdermal irrigation, central and peripheral body temperature, etc.). To the student will be evaluates his capacity to structure and analyze graphs. It is concluded that this practice allows to demonstrate diverse concepts of integration and physiological adaptation.

Keywords: integrative physiology; integrative biology; exercise physiology

\section{Resumo}

O objetivo desta experiência foi para projetar uma prática para integrar, em uma única sessão, os diferentes sistemas fisiológicos estudados até esse momento, na assignatura de "Fisiologia e Fisiopatologia", do primeiro ano do Curso de Enfermagem. Portanto, a práticachamada "Testes funcionais de diferentes sistemas", deve servir comouma experiência integradora. Um protocolo de exercício contínuo foi utilizado para medir a adaptação ao estresse em um período de tempo relativamente curto. Este tipo de

protocolo é semelhante ao utilizado pelo atleta em seus exercícios e permite a observação de alterações adaptativas ao estresse em diferentes variáveis fisiológicas (freqüência cardíaca, freqüência respiratória, ácido lático, glicemia, irrigação subdérmica, temperatura corporal central e periférica, etc.). No aluno é avaliada a capacidade de estruturar e analisar gráficos. Conclui-se que esta prática permite demonstrar diversos conceitos de integração e adaptação fisiológica.

Palavras-chave: fisiologia integrativa; biologia integrativa; fisiologia do exercício

\section{Introducción}

En el prólogo, escrito por el naturista y fisiólogo francés, Henry Cardot (1886-1942), del clásico libro de prácticas de fisiología de Jullien (1935), señala que las prácticas deben formar la culminación y la ilustración de las clases magistrales. Ciertamente, el aprendizaje en laboratorio deja una viva impresión en los alumnos al experimentar y observar los hechos que se analizan, así como también se adquiere la disciplina necesaria para la investigación (Cannon, 1929). Entre las nuevas áreas de la investigación biológica encontramos la genómica funcional, la biología de los sistemas y la biología integradora; todas ellas forman campos fundamentales de la fisiología (Silverthorn, 2014). El objetivo de esta experiencia fue integrar en una sola práctica los 
diferentes sistemas fisiológicos estudiados en las clases magistrales y en las prácticas de laboratorio de la asignatura de 'Fisiología y fisiopatología’, de primer año del Grado en Enfermería.

\section{Contextualización}

Después del proceso de transformación de los Diplomados y Licenciaturas a los Grados, se promovieron adaptaciones importantes en la teoría y en la práctica. Formando parte del Plan de Estudios del Grado en Enfermería, se encuentra la asignatura de formación básica, anual, de primer año, con 9 créditos totales. El curso comprende 14 sesiones de prácticas, que se realizan en el laboratorio o se desarrollan como modelos fisiológicos en el aula de informática. Diez de esas prácticas son de fisiología. La última práctica, de la parte de fisiología, denominada "Pruebas funcionales de diferentes sistemas", debe permitir estructurar una experiencia integradora de los diferentes sistemas fisiológicos ya desarrollados en la asignatura.

\section{Diseño y desarrollo}

En este contexto, fue donde se buscó y diseño una experiencia de laboratorio que permitiera que los alumnos pudieran observar el concepto de las funciones integradas y de los procesos (Silverthorn, 2014) que realiza nuestro organismo para la manutención de los valores internos; la homeostasia, pilar de la fisiología moderna (Davies, 2016).

¿Qué otro proceso más integrador existe que enfrentar a un sujeto a una situación de estrés? Sabemos que el ejercicio físico es una actividad estresante. Por ello, se utilizó como base de esta práctica un protocolo de ejercicio continuo, ya que permite que el sujeto adapte las diferentes variables posibles de analizar en un período relativamente corto de tiempo. En estas circunstancias, los sistemas biológicos deben estar en continua adaptación y el ejercicio o el entrenamiento, demuestran ser ejemplo de típicas respuestas de adaptación (Davies, 2016). Un ejercicio continuo permite una serie de ventajas para el control, seguimiento y valoración de la prueba realizada durante la práctica:

a) Permite un control más cuidadoso de muchas variables.

b) No pone en riesgo al sujeto, ya que siempre se trabaja con deportistas a una intensidad normalmente cercana a las del entrenamiento. 
c) Facilita evaluar y observar, en un sujeto sano, las adaptaciones del sistema cardiovascular y respiratorio, como el aumento de la frecuencia cardíaca y de la frecuencia respiratoria, que son de llamativo ejemplo para el alumno.

d) Permite dar respuesta directa, sobre conceptos metabólicos y del uso de la energía por las vías aeróbicas y anaeróbicas, al medir la progresión de los valores de ácido láctico durante el ejercicio.

e) Entrega la posibilidad de observar las modificaciones de la glucemia, en especial durante los primeros 10 minutos de ejercicio, como un ejemplo del balance entre las funciones digestivas, pancreáticas y hepáticas.

f) Permite el uso de cámaras térmicas, para que el alumno observe los enormes cambios en la irrigación sanguínea subdérmica, entre el reposo y la actividad, que dan ejemplo de los innumerables procesos de adaptación para controlar la temperatura interna.

g) Y, sobre este complejo adaptativo, las modificaciones de la temperatura timpánica y de zonas del rostro y del cuerpo, permiten al alumno comprender con claridad el sistema de la regulación térmica; en especial, cuando se observan los cambios bruscos de estas temperaturas y el sujeto que se ejercita, inicia la explosión de la sudoración.

h) Finalmente, permite tantas opciones como la diversidad de métodos de análisis que cuente el propio laboratorio.

\section{Evaluación}

La experiencia es evaluada por la capacidad de análisis y de asociación de las diferentes variables observadas en la práctica, al compararlas con los conceptos adquiridos en las clases magistrales de los diferentes sistemas fisiológicos estudiados durante el curso. El alumno debe estructurar gráficos y analizarlos, intentando, con los conocimientos ya adquiridos, dar respuesta a las adaptaciones observadas.

\section{Conclusiones}

Ciertamente, la experiencia nos muestra que es una de las prácticas más ilusionantes para el alumno. Qué permite demostrar conceptos como la homeostasis, la alostasis, la 
heterostasis, la hormesis, etc., en los cortos tiempos de adaptación que pueden permitir dos horas, de una sesión de prácticas.

\section{Referencias}

Cannon, W. B. (1929). Curso de fisiología de laboratorio. $1^{a}$ ed. española. New York: D. Appleton and Company.

Davies, K. J. A. (2016). Adaptative homeostasis. Mol Aspects Med, 49, 17.doi.org/10.1016/j.mam.2016.04.007

Jullien, A. (1935). Travaux practiques de physiologie et príncipes d'experimentation. Paris: Baillière et Fils.

Silverthorn, D. U. (2014). Fisiología humana: un enfoque integrado. $6^{\mathrm{a}}$ ed. México: Panamericana. 